\title{
Magneto-optic Kerr effect in a spin-polarized zero-moment ferrimagnet
}

\author{
Karsten Fleischer, ${ }^{1,2}$ Naganivetha Thiyagarajah, ${ }^{3}$ Yong-Chang Lau, ${ }^{3}$ Davide Betto, ${ }^{3}$ Kiril Borisov, ${ }^{3}$ Christopher C. Smith, ${ }^{1}$ \\ Igor V. Shvets, ${ }^{1}$ J. M. D. Coey, ${ }^{3}$ and Karsten Rode ${ }^{3}$ \\ ${ }^{1}$ School of Physics, Trinity College Dublin, College Green, Dublin 2, Ireland \\ ${ }^{2}$ School of Physical Sciences, Dublin City University, Dublin 9, Ireland \\ ${ }^{3}$ Centre for Research on Adaptive Nanostructures and Nanodevices, and School of Physics, Trinity College Dublin, \\ College Green, Dublin 2, Ireland
}

(Received 21 June 2018; revised manuscript received 3 September 2018; published 26 October 2018)

\begin{abstract}
The magneto-optical Kerr effect (MOKE) is often assumed to be proportional to the magnetization of a magnetically ordered metallic sample; in metallic ferrimagnets with chemically distinct sublattices, such as rare-earth transition-metal alloys, it depends on the difference between the sublattice contributions. Here we show that in a highly spin polarized, fully compensated ferrimagnet, where the sublattices are chemically similar, a signal is observed even when the net moment is negligible. We analyze the spectral ellipsometry and MOKE of $\mathrm{Mn}_{2} \mathrm{Ru}_{x} \mathrm{Ga}$ and show that this behavior is due to a highly spin-polarized conduction band dominated by one of the two manganese sublattices $(4 c)$ which creates helicity-dependent reflectivity dominated by a broad Drude tail. Our findings open prospects for studying spin dynamics in the infrared.
\end{abstract}

DOI: 10.1103/PhysRevB.98.134445

\section{INTRODUCTION}

Spin valves, where angular momentum can be transferred from a reference layer to a free layer, are expected to form the basis of future memory devices and oscillators operating in the far infrared frequency domain [1]. The key materials properties necessary to achieve these frequencies are a high Fermi-level spin polarization $P$, low net magnetization $M_{s}$, and a high effective magnetic anisotropy field $\mu_{0} H_{\mathrm{k}}$. A compensated ferrimagnetic half metal where negligible magnetization can be obtained, coupled with full $(P=100 \%)$ spin polarization, would be an ideal choice [2].

Such a material was suggested by van Leuken and de Groot [3] in 1995, but the first experimental evidence was only provided by the work of Kurt et al. [4] in 2014. We have recently shown that the material, $\mathrm{Mn}_{2} \mathrm{Ru}_{x} \mathrm{Ga}$ (MRG), shows negligible $M_{s}$ over a wide temperature and thermal treatment range [5,6], high spin polarization [4] as reflected in the anomalous Hall effect [7], produces sizable magnetoresistive effects in spin electronic structures [8] such as magnetic tunnel junctions [9], and proposed an ab initio model reconciling the experimental observations with density functional theory (DFT) band structure calculations [10].

Time-resolved magneto-optical Kerr/Faraday effect is particularly suited to determine the Larmor frequency of thin-film samples, especially when the resonance frequency exceeds the experimental range of cavity- or network-analyzer-based techniques. The unique properties of MRG indicate that the material may be a candidate for low-power all-optical switching as the magnetic compensation temperature $T_{\text {comp }}$ can be tuned to lie just above room temperature [11], but these measurements on new materials are complicated by the lack of an accepted, wavelength-dependent optical model.

Ferrimagnets with two chemically distinct sublattices, notably the rare-earth transition metal alloys, have been investi- gated by spectroscopic MOKE $[12,13]$. The spectral response of the $4 f$ and $3 d$ elements allow the temperature dependence of the Kerr effect of each sublattice to be determined. The aspect we present here is that in a ferrimagnet with chemically identical, but structurally inequivalent, sublattices it is possible to track the spin polarization associated with only one of the sublattices.

We first show that even at magnetic compensation, MRG exhibits a clear Kerr rotation $\left(\theta_{K}\right)$. Spectroscopic ellipsometry, in combination with the spectroscopic polar MOKE [14], allow us to determine the diagonal $\left(\tilde{\varepsilon}_{x x}\right)$ and off-diagonal $\left(\tilde{\varepsilon}_{x y}\right)$ dielectric tensor components as a function of Ru concentration $x$. Polar MOKE is often used simply to measure hysteresis loops using monochromatic light, and the spectroscopic variant provides more specific information on the sites responsible for the magnetic response. Our measurements show a clear correlation between the infrared Kerr rotation and the electrically-measured Hall angle, providing contactless, optical measurements of this quantity. The experimentally determined, optical model developed here will facilitate measurements in the time and frequency domains that can advance the development of spin electronic devices operating in the far infrared frequency range.

\section{SAMPLE DETAILS}

The thin-film samples studied here were grown on $\mathrm{MgO}$ (001) substrates by dc magnetron sputtering at $250^{\circ} \mathrm{C}$ substrate temperature in a 'Shamrock' system with a base pressure of $2 \times 10^{-8}$ Torr. The films were co-sputtered from Ru and $\mathrm{Mn}_{2} \mathrm{Ga}$ targets. The $\mathrm{Ru}$ concentration was controlled by varying the $\mathrm{Mn}_{2} \mathrm{Ga}$ target plasma power while keeping that of the Ru fixed. The samples were then capped with a protective $2 \mathrm{~nm}$ layer of amorphous aluminium oxide. The 


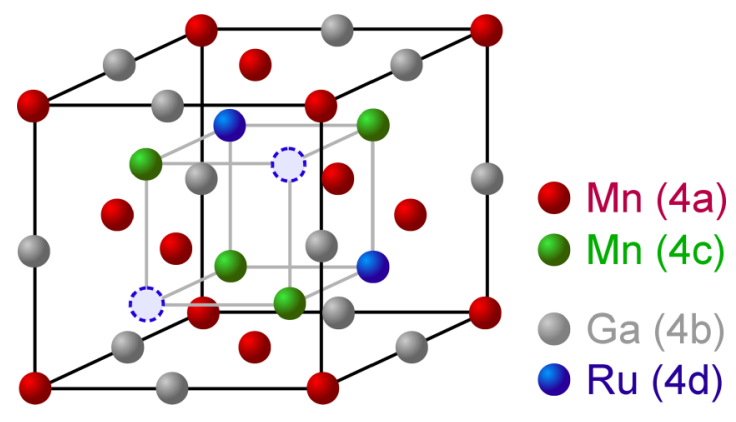

FIG. 1. Schematic of the MRG crystal structure. There are three filled fcc sublattices [red: Mn site (4a), gray: Ga site (4b), green: Mn site $(4 c)$ ]. Ruthenium occupies the remaining fcc sublattice [blue: $\mathrm{Ru}$ site $(4 d)]$. The two Mn sites are nonequivalent in the structure as the $4 a$ site has only $\mathrm{Ga}$ as nearest neighbor, while the $4 c$ site $\mathrm{Ru}$, or vacancies (dashed circles) depending on the Ru content $x$.

crystal structure, lattice parameters, and strain of the films were characterized by $2 \theta-\theta$ x-ray diffraction and reciprocal space mapping using a Bruker D8 Discover instrument, with a monochromatic copper $\mathrm{K}_{\alpha 1}$ source. We found that the in-plane lattice constant $a$ is $0.5956 \mathrm{~nm}=\sqrt{2} a_{\mathrm{MgO}}$ for all samples, indicating that MRG grows epitaxially on $\mathrm{MgO}$ with its basal plane rotated by $45^{\circ}$ with respect to the substrate. The out-of-plane parameter $c$ varies between $0.598 \mathrm{~nm}$ and $0.618 \mathrm{~nm}$, depending on $\mathrm{Ru}$ concentration and film thickness $(\sim 26$ to $81 \mathrm{~nm})$. The measurement confirms a slight, substrate-induced, tetragonal distortion of the cubic structure. An illustration of the crystal is given in Fig. 1.

In order to determine the Ru concentration, we deposited four MRG samples with varying $\mathrm{Mn}_{2} \mathrm{Ga}$ target power and a pure $\mathrm{Ru}$ film. The density and thickness were then measured using $\mathrm{x}$-ray reflectivity (XRR). Based on the measured density and lattice parameters of these five control samples, we established a relation between $\mathrm{x}$-ray density and $\mathrm{Ru}$ concentration $x$, against which all the samples were calibrated. The transport properties of the thin films were measured on nonpatterned films in a Quantum Design physical properties measurement system (PPMS) at temperatures ranging from 10 to $400 \mathrm{~K}$ in an applied magnetic field, $\mu_{0} H$, of up to $12 \mathrm{~T}$.

\section{EXPERIMENTAL DETAILS}

Both ellipsometry and magneto-optical Kerr spectroscopy were used to determine the optical and magneto-optical properties of the films. Spectroscopic MOKE was recorded at nearnormal incidence with an in-house built reflectance anisotropy spectrometer, based on the design of Aspnes [15]. Light from a Xe lamp passes through a Rochon $\mathrm{Mg}_{2} \mathrm{~F}$ polarizer and is reflected from the sample. The beam then passes through a photoelastic modulator (PEM), an analyzing polarizer, and a monochromator before finally reaching a diode detector system. All measurements were performed from 0.35 to $5 \mathrm{eV}$, using a Bentham TMc300 triple-grating monochromator and three individual photodiodes (InAs, InGaAs, $\mathrm{Si}$ ). The procedure for extracting the MOKE rotation has been discussed elsewhere [16-18]. All samples were measured at room temperature in remanence (no external field). Samples were magnetized before these measurements in fields of up to
$12 \mathrm{~T}$ at room temperature. Selected samples have also been magnetized at $200 \mathrm{~K}$.

To correct for any setup or nonmagnetic anisotropy, we recorded spectra for two orthogonal in-plane directions as well as for samples magnetized in opposite directions. The spectra obtained by reversal of $\vec{M}$, were strictly equal to those derived from a rotation of the sample of $90^{\circ}$ around the optical axis. Due to larger errors in the Kerr-ellipticity measurement in a PEM setup without in situ magnetization reversal, only the Kerr rotation $\left(\theta_{K}\right)$ will be discussed.

A Sopra GESP 5 spectroscopic ellipsometer was used over an energy range from 1.5 to $5 \mathrm{eV}$ with incidence angles of $70^{\circ}, 75^{\circ}$, and $80^{\circ}$. Both components of the thin film dielectric function $\left(\tilde{\varepsilon}_{x x}, \tilde{\varepsilon}_{x y}\right)$, were derived from the raw measurements by minimizing the deviation between modelled and measured $\theta_{K}$ as well as ellipsometric data $(\tan \Psi, \cos \Delta)$ over the whole spectral range. Full transfer matrix calculations of the air/AlO $x$ /MRG/MgO layer stack have to be evaluated, as in contrast to simple bulk samples no analytical expression for the relationship between measured $\tan \Psi, \cos \Delta$, and $\theta_{K}$ exists. The spectral shape of $\tilde{\varepsilon}$ was simulated by a standard Drude-like component plus three harmonic oscillators (Drude-Lorentz oscillator). To reduce uncertainties in $\tilde{\varepsilon}_{x x}$, only the MRG layer was modelled. The sample thickness and interface roughness, as measured by XRR was used as input parameters together with the known bulk dielectric functions of the $\mathrm{MgO}$ substrate and the $\mathrm{AlO}_{x}$ capping layer.

In thin film structures, the $\theta_{K}$ spectra depend not only on the off-diagonal dielectric tensor components $\tilde{\varepsilon}_{x y}=i Q m_{z} \tilde{\varepsilon}_{x x}$ of the magnetic material but also on the overall reflectance of the stack. Knowing the diagonal components $\tilde{\varepsilon}_{x x}$ from the ellipsometric model, the measured $\theta_{K}$ spectral signature can then also be modelled, and hence $\tilde{\varepsilon}_{x y}$ determined using the same methodology without skewed results caused by variations in $\tilde{\varepsilon}_{x x}$ and sample thickness/roughness and modulation of the overall reflectivity [19-21].

For all optical models, the MRG and $\mathrm{AlO}_{x}$ thicknesses determined by XRR were treated as fixed parameters. All interfaces have been treated as sharp, while the dielectric function of the $\mathrm{AlO}_{x}$ coating has been modelled by a Bruggeman effective medium of crystalline $\mathrm{Al}_{2} \mathrm{O}_{3}$ and air with a fill factor set to the density ratio of the XRR fitted capping layer and crystalline $\mathrm{Al}_{2} \mathrm{O}_{3}$. We treat the MRG to be perfectly cubic, as the deviation from cubic symmetry due to epitaxial strain of $\sim 1 \%$ [4] is well below the sensitivity of our instrument.

\section{MOKE: DIRECT MEASUREMENTS}

Due to the different crystallographic sites occupied by the two magnetic sublattices in ferrimagnets, the temperature dependence of the magnetization of the two sublattices differ. Generally, perfect compensation and zero net moment occurs only at a specific temperature $\left(T_{\text {comp }}\right)$ where the magnitudes of the two magnetic sublattices are equal but opposite in sign. For MRG this temperature depends on both Ru content $x$ and the biaxial substrate-induced strain.

Upon crossing $T_{\text {comp }}$ in zero applied field the net magnetization changes sign whereas the spin-dependent DOS remains unchanged because the individual sublattices have not changed direction. We therefore expect that the sign of 


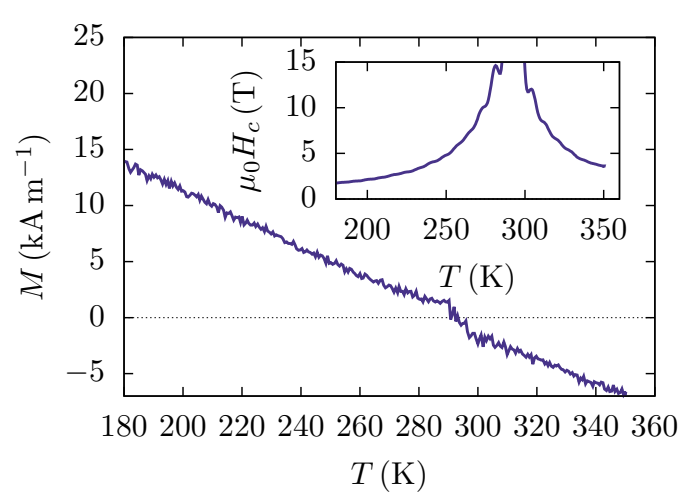

FIG. 2. SQUID magnetometry of sample with $T_{\text {comp }}$ close to RT. The net magnetization of the sample was measured at remanence on warming after saturation at low temperature. In the inset we show an estimate of the coercive field, $\mu_{0} H_{c}$, as a function of temperature. Around room temperature, even $15 \mathrm{~T}$ is insufficient to saturate the magnetization.

any property that depends on the spin-resolved DOS such as MOKE, tunnel magnetoresistance (TMR), and anomalous Hall effect, will exhibit opposite signs depending on whether the sample was magnetized below or above $T_{\text {comp }}[7,9]$.

In Fig. 2 we show the temperature dependence of the net magnetic moment of an MRG sample with $T_{\text {comp }} \sim 295 \mathrm{~K}$. The inset gives an estimate of the coercive field as a function of temperature. Close to $T_{\text {comp }}$, the anisotropy field $\mu_{0} H_{k}$ diverges, and as the coercive field is directly related to the anisotropy field, the sample cannot be magnetically saturated in this temperature range. We now compare MOKE spectra of two samples with $T_{\text {comp }}$ of $295 \mathrm{~K}$ and $250 \mathrm{~K}$, respectively. The data are shown in Fig. 3. When magnetised at RT, i.e., above $T_{\text {comp }}$, both samples exhibit clear MOKE negative in sign. The sample with $T_{\text {comp }}$ closest to RT appears to have

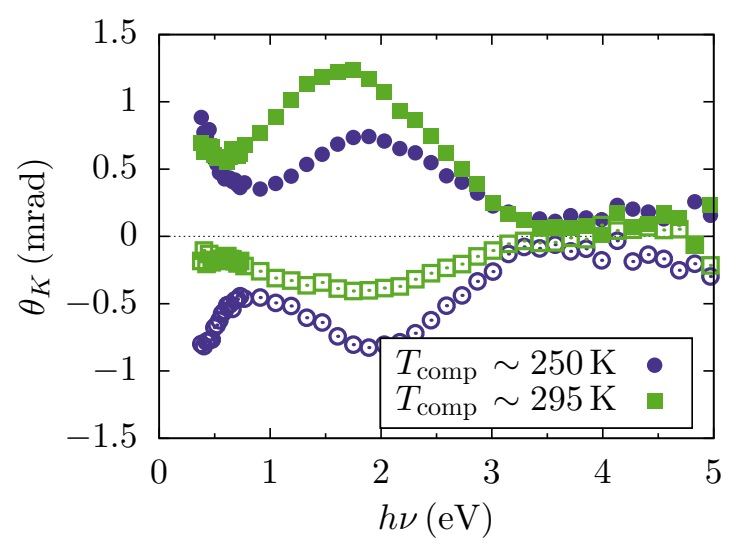

FIG. 3. Room-temperature Kerr rotation as a function of photon energy for samples with $T_{\text {comp }} \sim 295 \mathrm{~K}$ and $250 \mathrm{~K}$, respectively. The samples were first magnetized at RT in a field of $\mu_{0} H=2 \mathrm{~T}$ (above $T_{\text {comp }}$, open symbols). Subsequently, the samples were remagnetized, this time well below $T_{\text {comp }}\left(T_{\text {sat }}=200 \mathrm{~K}\right.$, filled symbols). Although all measurements are done at RT, the sign of the MOKE signal is reversed depending on whether magnetization is carried out above or below $T_{\text {comp }}$. When $T_{\text {comp }} \sim 295 \mathrm{~K}$, full saturation is only achieved when magnetized well below $T_{\text {comp }}$. a reduced magnitude. We then magnetize both samples well below their respective $T_{\text {comp }}, T_{\text {sat }}=200 \mathrm{~K}$, and subsequently warm to RT in zero applied field. This procedure ensures that while the net magnetic moment changes sign when crossing $T_{\text {comp }}$, the spin-dependent density of states (DOS) remains unchanged. We then record MOKE spectra at RT. As can be seen from Fig. 3, both samples exhibit a change of sign as expected, and crucially, the sample with $T_{\text {comp }} \sim 295 \mathrm{~K}$ and thus near zero net moment now displays as strong or stronger MOKE signal as the sample measured further away from $T_{\text {comp. }}$. This set of measurements proves that MOKE in MRG depends on the spin-dependent DOS and not the net magnetic moment, and furthermore even when the net magnetic moment is negligible, the MOKE signal is clearly observed.

We note here that disproportionality between the remnant magnetization and the polar MOKE measured during a hysteresis loop have been observed previously, in different circumstances [22,23]. This was attributed to coupling, during switching, of the in-plane magnetization and the polar MOKE through higher order terms. It was observed in highly anisotropic exchange-biased materials and only during switching. The samples measured here do not exhibit uniaxial in-plane anisotropy, and they are all measured at remanence. In order to pinpoint the origin of MOKE in MRG, we recorded angle and energy dependent ellipsometry, which in combination with the measured Kerr rotation allow us to determine all matrix elements of the dielectric tensor.

\section{OPTICAL MODEL}

We have shown that MOKE is not determined by the net magnetic moment but depends on the spin dependent DOS in MRG. MOKE measurements alone are insufficient to determine $\tilde{\varepsilon}_{x x}$ and $\tilde{\varepsilon}_{x y}$. We therefore first use spectroscopic ellipsometry to determine $\tilde{\varepsilon}_{x x}$ and subsequently develop the optical model including the off-diagonal tensor elements $\tilde{\varepsilon}_{x y}$ for MRG.

In Fig. 4 we show the dielectric function $\tilde{\varepsilon}_{x x}$ as extracted from least square fits of the measured $\tan \Psi$ and $\cos \Delta$ for a set of samples with varying Ru concentration $x$. Our optical model, used for both $\tilde{\varepsilon}_{x x}$ and $\tilde{\varepsilon}_{x y}$, is based on the following considerations:

For metallic materials such as MRG the dielectric response is usually dominated by a strong Drude-like tail caused by free electron absorption, also known as intraband transitions [25]. This contribution is the reason why the imaginary part of $\tilde{\varepsilon}_{x x}$ of MRG does not tend towards zero $\left(\sigma \sim 45 \times 10^{14} \mathrm{~s}^{-1}\right.$ at $h v=1 \mathrm{eV}$ ) in the infrared region. Furthermore, there are absorption features in the visible (VIS-peaks $\sim 2.3 \mathrm{eV}$ ) which we attribute to interband transitions on both Mn sites, ultraviolet $(\mathrm{UV} \sim 3.2 \mathrm{eV}$ ) due to $\mathrm{Ga}$, and a deep ultraviolet contribution (DUV $>5.5 \mathrm{eV}$ ) necessary to account for the line shape but that cannot be assigned to a specific element. The assignment of the VIS structure to Mn interband transitions is motivated by several experimental and theoretical findings, including similar spectral features in bulk $\alpha-\mathrm{Mn}$ [24], a strong peak in the unoccupied density of states in ab initio calculations for bulk Mn [26], and finally the similarity in the shift of the energetic position of the VIS peak with increasing 

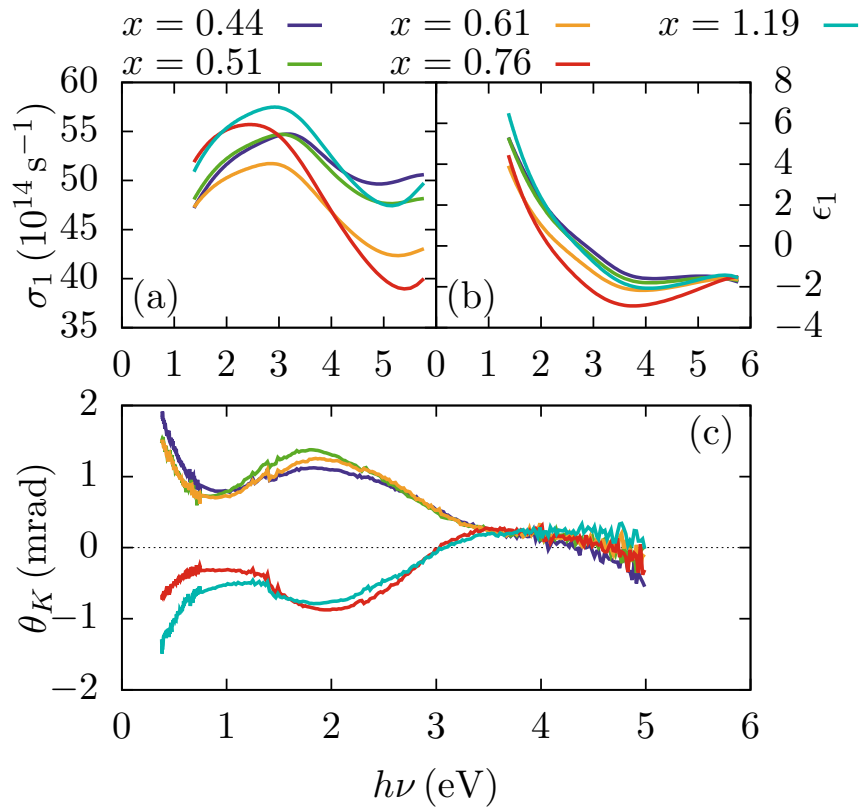

FIG. 4. (a) and (b): Imaginary and real components of the diagonal tensor elements $\tilde{\varepsilon}_{x x}$ of the dielectric function of MRG as a function of photon energy and $\mathrm{Ru}$ concentration $x$. The imaginary part is shown in terms of optical conductivity $\left(\sigma_{1}=\varepsilon_{2} \omega / 4 \pi\right)$ [24], to highlight the small differences between samples. The behavior is dominated by the Drude-like metallic response, with smaller deviations caused by interband transitions. (c) MOKE spectra for the same series of samples used to infer the off-diagonal $\tilde{\varepsilon}_{x y}$ tensor elements.

$\mathrm{Ru}$ concentration, following the same trend as that seen in site-selective XAS/XMCD measurements on $\mathrm{Mn}_{2} \mathrm{Ru}_{x} \mathrm{Ga}$ [5]. To minimize the number of free parameters, we therefore describe the diagonal part of the dielectric function via one Drude-like oscillator at $\omega=0 \mathrm{eV}$, and three broad harmonic oscillators $(\Gamma>6 \mathrm{eV})$ in the VIS, UV, and DUV ranges, respectively.

The Kerr spectra show strong features in the IR and the VIS regions, associated with the Drude-tail and the Mn, respectively, but no separate features in the UV and DUV. As the magnetism in MRG is dominated by Mn in two antiferromagnetically coupled sublattices, $\tilde{\varepsilon}_{x y}$ can be described in more detail. We associate the single, broad, VIS oscillator observed in ellipsometry at $h v \sim 2.3 \mathrm{eV}$ with two individual components in the Kerr spectra (NIR and VIS in Fig. 5, bottom panel), which we assign to the spin-dependent transitions on the Mn- $4 c$ and Mn- $4 a$ site. In agreement with our assignment above of the UV and DUV transitions to nonmagnetic components, no separate UV and DUV oscillators are required to describe $\tilde{\varepsilon}_{x y}$.

This assignment is further motivated by our earlier x-ray absorption measurements [5] where we find that the final states of the $2 p \rightarrow 3 d$ transitions of $\mathrm{Mn}$ in the two sites are only separated by approximately $1 \mathrm{eV}$, and no circular magnetic dichroism on the $\mathrm{Ga} L_{2,3}$ edges nor on the $\mathrm{Ru} M_{4,5}$ edges, as well as the dielectric functions of Mn [24] and Ga [27].

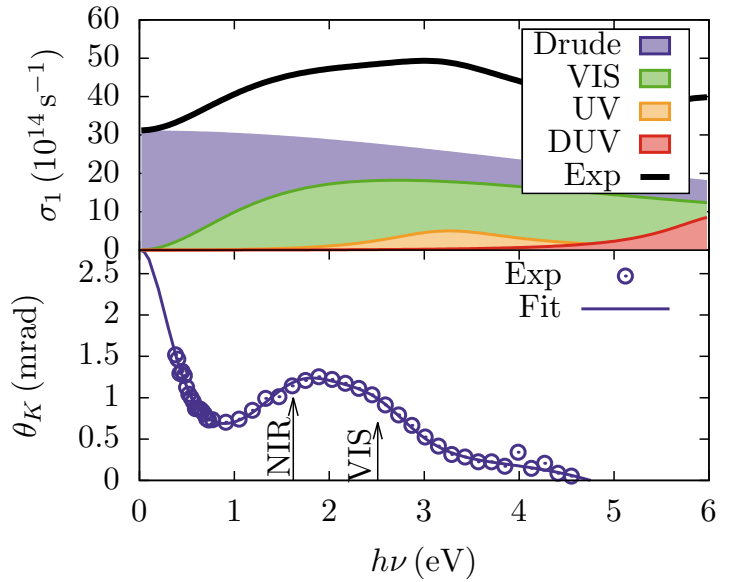

FIG. 5. Complete optical model and experimental data for an MRG sample with $x=0.61$. (Top) Experimentally observed optical conductance (line) and the fitted contributions from the four harmonic oscillators (areas) described in the text. (Bottom) Experimental Kerr rotation (points) and fit to the data (line) based on the optical model described in the main text.

In Fig. 5 we show an example of a fit to the model described above. Having established the diagonal tensor elements $\tilde{\varepsilon}_{x x}$ we then model the off-diagonal terms which we plot as the expected Kerr rotation $\theta_{K}$ (the real part of the complex Kerr rotation). The agreement between the experimental data and the optical model is excellent, indicating that our model is well justified as well as highlighting the predominant role of $\mathrm{Mn}$ in MRG magnetization. The (extrapolated) steep increase in MOKE in the IR and towards DC ( $h v \rightarrow 0 \mathrm{eV})$ is clearly an effect of the highly spin-polarized conduction band, as reflected by the Drude contribution to both diagonal and offdiagonal tensor elements.

Our modeling allows us to study in finer detail the behavior of the spectral components of the ellipsometry and MOKE as a function of Ru concentration $x$ (Fig. 4). When the Ru concentration $x$ changes from 0.44 to 1.19 , the oscillator associated with Ga (UV) changes position only slightly from 3.4 to $3.2 \mathrm{eV}$ whereas the oscillator associated with Mn (VIS), exhibits a stronger trend and moves from 3.0 to $2.2 \mathrm{eV}$ with increasing $x$. With increasing Ru content, the electronic occupation of $\mathrm{Mn}$ is increasing linearly, wheres that of Ga remains constant. The (small) changes associated with Ga are thus due to increasing tetragonality of the crystal structure while those associated with Mn correlate directly with the band filling. As described above, the MOKE in MRG is dominated by the contributions of $\mathrm{Mn}$, both through the spin polarisation of the conduction band as well as single-ion transitions. The two oscillators used to describe these transitions in MOKE can therefore be associated with $\mathrm{Mn}$ in the $4 c$ position ( $1.7 \mathrm{eV}-\mathrm{NIR})$ and in the $4 a$ position $(\sim 2.5 \mathrm{eV}-\mathrm{VIS})$. Due to the half-metallic nature of the sample, when Ru content is increased, the electronic occupation of $\mathrm{Mn}$ in the $4 c$ position increases, leading to a blueshift (from 1.4 to $1.8 \mathrm{eV}$ ) of the oscillator associated with this position. Simultaneously, adding Ru decreases the spin-gap [10] thereby inducing a red shift (from 2.7 to $2.4 \mathrm{eV}$ ) of the oscillator associated with $\mathrm{Mn}$ in the $4 a$ position. More details on the optical model 


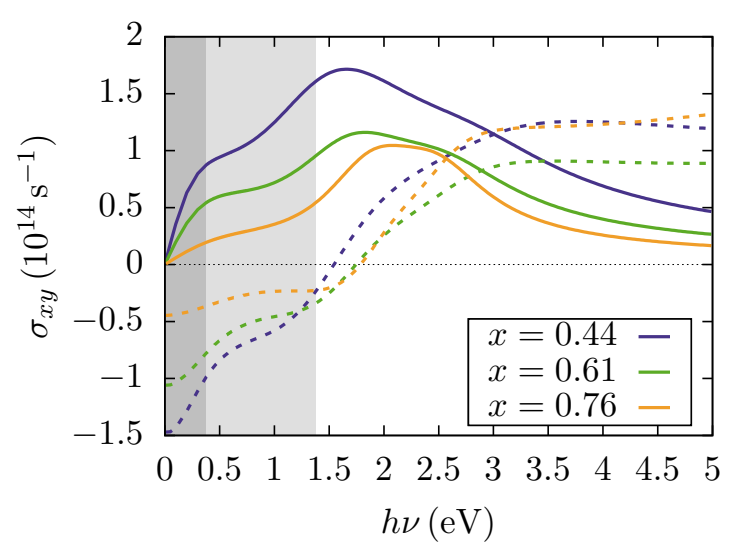

FIG. 6. $\sigma_{x y}$ extracted from the optical model for three different Ru concentrations $x$. The real and imaginary parts are plotted in solid and broken lines, respectively. In the light and dark gray areas, the curves are extrapolated from the experimentally available range in ellipsometry and MOKE, respectively. Please not that $\operatorname{Im}\left(\sigma_{x y}\right)$ is only determined up to a potential integrational constant.

parameter as function of Ru concentration $x$ are found in the Supplemental Material [28].

In Fig. 6 we show the off-diagonal component of the dielectric tensor as a function of photon energy, extracted from the fit of the experimental ellipsometry and MOKE data, in terms of $\sigma_{x y}$. Unlike the raw MOKE spectra, differences between different compositions are now clearly visible, and highlight, independently of our discussion focused on MRG, the need to employ robust optical modeling when reporting MOKE data on thin film samples of even slightly varying thickness.

We finally turn to a comparison of the DC limit of the magneto-optical properties with the anomalous Hall angle measured by standard electronic magnetotransport. As outlined above, our optical model describes the behavior of MRG fully in the spectral region measured, and we therefore expect that extrapolation outside this region should accurately predict

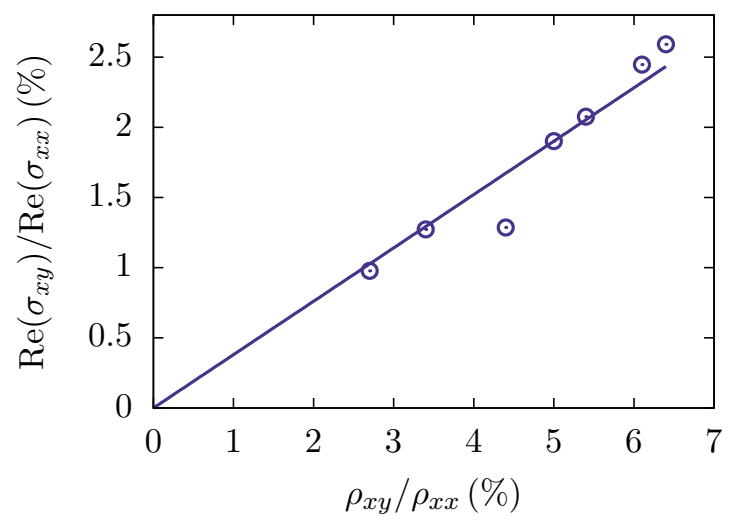

FIG. 7. Ratio of the real part of the off-diagonal and on-diagonal terms of the dielectric tensor extrapolated to $0.4 \mathrm{eV}$ plotted as a function of the electrically measured anomalous Hall angle. The straight line is a fit to the data and provides a guide to the eye. As expected, the DC limit of the magneto-optical characterization agrees very well with the values obtained through electronic transport measurements. the behavior at different photon energies. The lower limit is of course DC where optical measurements are not possible, but where we can record magnetotransport, in particular anomalous Hall effect [7]. The ratio $\rho_{x y} / \rho_{x x}$ is the Hall angle, the DC limit of the ratio $\operatorname{Re}\left(\sigma_{x y}\right) / \operatorname{Re}\left(\sigma_{x x}\right)$. In Fig. 7 we plot the ratio determined from the extrapolation of our optical model as a function of the Hall angle determined by magnetotransport measurements as described in II. The ratio was evaluated at $h v=0.4 \mathrm{eV}$ as we only need to extrapolate $\sigma_{x x}$ and not $\sigma_{x y}$ at this energy. As expected we observe a strong correlation between the two measurements, most certainly because the two effects share a common origin; the high spin polarization of the conduction band. The deviations from a perfectly proportional behavior is due to small differences in the scattering matrices that have a big influence in transport measurements whereas they do not influence the values obtained by optical means. We also emphasize that whereas electronic transport probes the entire thickness of a metallic film, MOKE and ellipsometry only probe the skin-depth, which varies with photon energy, found here to be $5 \mathrm{~nm}$ at $2 \mathrm{eV}$ and $40 \mathrm{~nm}$ at $0.4 \mathrm{eV}$.

\section{CONCLUSION}

We have shown that MOKE in zero-moment half metals persists, even when the net magnetic moment crosses zero, and that its origin is dominated by the highly spin-polarized conduction band associated in MRG with Mn in the $4 c$ site [5]. The sign of the MOKE depends on whether the sample was magnetised above or below its compensation temperature, as the process of magnetization sets the direction of the axis of quantization for the spin-polarized density of states.

By combining spectroscopic ellipsometry and MOKE it was possible to construct an optical model that identifies the optically active components of MRG, and we were able to use this to infer the full parameterized dielectric tensor as a function of photon energy in the range 0 to $5 \mathrm{eV}$. The model is deliberately designed to minimize the number of free parameters, yet it captures with reasonable accuracy both the nonmagnetic and the magnetic contributions for MRG in the spectral range measured. Based on the model, we compare the results obtained by optical means with the anomalous Hall angle recorded by electronic transport measurements. Although the DC limit is far beyond our experimentally available range, the extrapolated values of $\sigma_{x y}$ agree remarkably well with those obtained by transport measurements.

Differences between magnetic properties observed by anomalous Hall effect and MOKE are often used nowadays as a fingerprint of topological electronic transport [29]. An extension of this study with a more in-depth comparison between the two in high and zero applied magnetic fields, may allow an unambiguous determination of the scattering coefficients that lead to topologically driven spin structures in for example noncollinear ferrimagnets, as a complete optical model is necessary to disentangle effects that depend solely on differences in sample thickness and reflectivity from those that are purely magnetic in nature. Finally, this work opens a path to study the behavior of magnetic domains in antiferromagnets by MOKE microscopy [30], while the shown 
spectral dependence highlights photon energies most suitable for pump-probe based time dependent measurements [31].

\section{ACKNOWLEDGMENTS}

This project has received funding from the European Union's Horizon 2020 research and innovation programme under Grant agreement No. 737038. K.F. and I.V.S. acknowledges support from Science Foundation Ireland (SFI), Grant No. 06/IN.1/I91. D.B. gratefully acknowledges funding from the Irish Research Council. This work was supported by SFI through AMBER and through Grant No. 16/IA/4534.
[1] D. Betto, K. Rode, N. Thiyagarajah, Y.-C. Lau, K. Borisov, G. Atcheson, M. Žic, T. Archer, P. Stamenov, and J. M. D. Coey, AIP Adv. 6, 055601 (2016).

[2] M. Hakimi, M. Venkatesan, K. Rode, K. Ackland, and J. M. D. Coey, J. Appl. Phys. 113, 17B101 (2013).

[3] H. van Leuken and R. A. de Groot, Phys. Rev. Lett. 74, 1171 (1995).

[4] H. Kurt, K. Rode, P. Stamenov, M. Venkatesan, Y.-C. Lau, E. Fonda, and J. M. D. Coey, Phys. Rev. Lett. 112, 027201 (2014).

[5] D. Betto, N. Thiyagarajah, Y.-C. Lau, C. Piamonteze, M.-A. Arrio, P. Stamenov, J. M. D. Coey, and K. Rode, Phys. Rev. B 91, 094410 (2015).

[6] K. E. Siewierska, G. Atcheson, K. Borisov, M. Venkatesan, K. Rode, and J. M. D. Coey, IEEE Trans. Magn. 53, 1 (2017).

[7] N. Thiyagarajah, Y.-C. Lau, D. Betto, K. Borisov, J. Coey, P. Stamenov, and K. Rode, Appl. Phys. Lett. 106, 122402 (2015).

[8] K. Borisov, G. Atcheson, G. D’Arcy, Y.-C. Lau, J. M. D. Coey, and K. Rode, Appl. Phys. Lett. 111, 102403 (2017).

[9] K. Borisov, D. Betto, Y.-C. Lau, C. Fowley, A. Titova, N. Thiyagarajah, G. Atcheson, J. Lindner, A. M. Deac, J. M. D. Coey, P. Stamenov, and K. Rode, Appl. Phys. Lett. 108, 192407 (2016).

[10] M. Žic, K. Rode, N. Thiyagarajah, Y.-C. Lau, D. Betto, J. M. D. Coey, S. Sanvito, K. J. O'Shea, C. A. Ferguson, D. A. MacLaren, and T. Archer, Phys. Rev. B 93, 140202 (2016).

[11] K. Vahaplar, A. M. Kalashnikova, A. V. Kimel, S. Gerlach, D. Hinzke, U. Nowak, R. Chantrell, A. Tsukamoto, A. Itoh, A. Kirilyuk, and T. Rasing, Phys. Rev. B 85, 104402 (2012).

[12] A. R. Khorsand, M. Savoini, A. Kirilyuk, A. V. Kimel, A. Tsukamoto, A. Itoh, and T. Rasing, Phys. Rev. Lett. 110, 107205 (2013).

[13] Y. Tsema, M. Savoini, A. Tsukamoto, A. V. Kimel, A. Kirilyuk, and T. Rasing, Appl. Phys. Lett. 109, 172403 (2016).

[14] J. Kerr, Phil. Mag., Series 5 3, 321 (1877).

[15] D. E. Aspnes and A. A. Studna, Phys. Rev. Lett. 54, 1956 (1985).
[16] K. Fleischer, R. Verre, O. Mauit, R. G. S. Sofin, L. Farrell, C. Byrne, C. M. Smith, J. F. McGilp, and I. V. Shvets, Phys. Rev. B 89, 195118 (2014).

[17] T. Herrmann, K. Lüdge, W. Richter, K. G. Georgarakis, P. Poulopoulos, R. Nünthel, J. Lindner, M. Wahl, and N. Esser, Phys. Rev. B 73, 134408 (2006).

[18] J. P. Cunniffe, D. E. McNally, M. Liberati, E. Arenholz, C. McGuinness, and J. F. McGilp, Phys. Stat. Sol. (b) 247, 2108 (2010).

[19] Y. V. Kudryavtsev, Y. P. Lee, and J. Y. Rhee, Phys. Rev. B 66, 115114 (2002).

[20] S. Halilov and E. Kulatov, J. Phys.: Condens. Matter 3, 6363 (1991).

[21] J. Zak, E. R. Moog, C. Liu, and S. D. Bader, Phys. Rev. B 43, 6423 (1991).

[22] R. M. Osgood, B. M. Clemens, and R. L. White, Phys. Rev. B 55, 8990 (1997).

[23] A. Tillmanns, S. Oertker, B. Beschoten, G. Güntherodt, C. Leighton, I. K. Schuller, and J. Nogués, Appl. Phys. Lett. 89, 202512 (2006).

[24] P. Johnson and R. Christy, Phys. Rev. B 9, 5056 (1974).

[25] P. Y. Yu and M. Cardona, Fundamentals of Semiconductors (Springer, Berlin, Heidelberg, 2005).

[26] D. Hobbs, J. Hafner, and D. Spišák, Phys. Rev. B 68, 014407 (2003).

[27] O. Hunderi and R. Ryberg, J. Phys. F 4, 2084 (1974).

[28] See Supplemental Material at http://link.aps.org/supplemental/ 10.1103/PhysRevB.98.134445 for more details on the influence of Ru doping $x$ on the optical properties.

[29] J. Matsuno, N. Ogawa, K. Yasuda, F. Kagawa, W. Koshibae, N. Nagaosa, Y. Tokura, and M. Kawasaki, Sci. Adv. 2, e1600304 (2016).

[30] K. E. Siewierska, N. Teichert, R. Schäfer, and J. M. D. Coey, IEEE Trans. Magn. 99, 1 (2018).

[31] N. Awari, S. Kovalev, C. Fowley, K. Rode, R. A. Gallardo, Y.-C. Lau, D. Betto, N. Thiyagarajah, B. Green, O. Yildirim, J. Lindner, J. Fassbender, J. M. D. Coey, A. M. Deac, and M. Gensch, Appl. Phys. Lett. 109, 032403 (2016). 